\title{
V. Le financement des politiques sportives locales : sport pour tous, sport professionnel, une dualité à réinventer
}

Dominique Charrier, Christophe Durand

\section{Citer ce document / Cite this document :}

Charrier Dominique, Durand Christophe. V. Le financement des politiques sportives locales : sport pour tous, sport professionnel, une dualité à réinventer. In: Annuaire des collectivités locales. Tome 25, 2005. Le financement des politiques locales. pp. 65-75;

doi : 10.3406/coloc. 2005.1840

http://www.persee.fr/doc/coloc_0291-4700_2005_num_25_1_1840

Document généré le 03/06/2016 


\title{
V. LE FINANCEMENT DES POLITIQUES SPORTIVES LOCALES : SPORT POUR TOUS, SPORT PROFESSIONNEL, UNE DUALITÉ À RÉINVENTER
}

\author{
Dominique CHARRIER et Christophe DURAND
}

La sphère publique, qu'elle soit nationale ou locale a assuré tout au long du $\mathrm{XX}^{\mathrm{e}}$ siècle un soutien massif au sport, aussi bien au niveau des pratiques du grand public qu'à celui du sport professionnel ${ }^{1}$. Si les dépenses sportives des pratiquants représentent $50 \%$ du total, depuis plusieurs décennies, les flux financiers publics issus des collectivités locales constituent un élément structurant du système avec $30 \%$ des dépenses ${ }^{2}$. Cette prédominance du secteur public s'inscrit toutefois dans un contexte marqué par une privatisation croissante d'une partie de l'offre sportive et par une professionnalisation des organisations qui gèrent le sport.

Plusieurs façons d'aborder les politiques sportives locales peuvent être envisagées par les observateurs. Certains auteurs ont analysé l'évolution des orientations politiques et des actions menées ${ }^{3}$, d'autres ont réalisé des monographies mettant en valeur l'importance des contextes locaux ${ }^{4}$, enfin des études macro-

1. J.-P. Clement, J. Defrance et C. Pociello, Sport et pouvoirs au Xxe siècle, Grenoble, Presses universitaires de Grenoble, 1994. P. ARNAun, « Pour une histoire des politiques sportives municipales », dans Le Sport et la ville, Spirales, $\mathrm{n}^{\circ}$ 5, 1992.

2. Les Chiffres-clés du sport, ministère de la Jeunesse, des Sports et de la Vie associative, 2004, disponibles sur le site <http://www.jeunesse-sports.gouv.fr/stats/chiffrescles2004.pdf>.

3. Par exemple, P. BAyeux, Le Sport et les collectivités territoriales, Paris, PUF, coll. «Que Sais-je ? ", n’ 3198, 1996. D. ChARRIER et C. DURAND (dir.), Le Financement du sport par les collectivités locales: des ambitions politiques aux choix budgétaires, Voiron, Presses universitaires du Sport, t. 2, 2002. M. Honta, Les Territoires de l'excellence sportive, Bordeaux, Presses universitaires de Bordeaux, 2002.

4. J.-P. CAllede et M. DANE, Sociologie des politiques sportives locales. Trente ans de vie sportive à Bègles, Bordeaux, MRSHA, 1991. 
économiques ${ }^{5}$ ont permis de caractériser durablement les dépenses sportives des collectivités locales. La synthèse qui suit emprunte aux trois types de démarche.

La distinction entre consommation active, «le sport qui se pratique », et consommation passive, « le sport qui se regarde », proposée par la Commission européenne structurera notre propos. Dans un premier temps, les évolutions des modes d'intervention des collectivités dans le cadre des activités sportives seront développées. Suivront les grandes tendances observées depuis une décennie en termes d'interventions publiques dans le sport professionnel.

\section{I) Financement des pratiques de loisir sportif: le rôle décisif des collectivités locales}

\section{1) Des données difficiles à constituer}

L'analyse macroéconomique du financement du sport par les collectivités locales a été engagée en 1990 . Une première étude ${ }^{6}$, à vocation exploratoire, a initié ce qui fut, pendant les années 1990 , un véritable observatoire du financement du sport par les collectivités locales. En effet, quatre études se sont succédé ${ }^{7}$, s'élargissant progressivement des conseils régionaux et généraux aux cent plus grandes villes (1992), puis aux communes de plus de 20000 habitants (1993) et, enfin, aux structures intercommunales les plus importantes (1995). Depuis 1998, l'étude macroéconomique, qui exigeait des moyens et des délais importants mais qui permettait une étude détaillée et méthodologiquement stable des financements locaux, a été remplacée par le calcul du seul total du budget sportif des collectivités locales, calcul réalisé, par extrapolation, par le ministère de la Jeunesse et des Sports.

5. N. Haschar-Noe, P. Bayeux, G. Klein et J. Vigneron, « Les politiques sportives et les services des sports des villes françaises de plus de 3000 habitants ", dans Sport, Europe, Stratégies, Revue européenne de management du sport, $\mathrm{n}^{\circ}$ 1, 1999. D. CHARRIER, (dir.), Le Financement du sport par les conseils régionaux, les conseils généraux, les communes de plus de 20000 habitants, les syndicats d'agglomération nouvelle et les communautés urbaines, Voiron, Presses universitaires du Sport, t. 1, 2002.

6. Voir Actes du colloque «Le financement du sport », Paris, ministère de la Jeunesse et des Sports, 1991.

7. Ces études ont été réalisées par le ministère de la Jeunesse et des Sports puis par le Centre d'études et de management de l'innovation sportive de l'université de Caen grâce à un financement du ministère de la Jeunesse et des Sports. Voir D. Charrier (2002), op. cit. 


\section{2) Des politiques sportives sous l'influence de la conjoncture économique et politique}

Ces études montrent d'abord que les politiques sportives locales ont un champ d'application qui dépasse largement les seules subventions aux clubs sportifs. Il convient de rappeler que, sur la période observée, les collectivités locales réservent près de la moitié de leurs dépenses aux équipements (investissement et fonctionnement), un quart aux dépenses de personnel et que le dernier quart couvre les subventions (dont $25 \%$ attribuées aux organisations sportives participant à des compétitions nationales et/ou internationales). En conséquence, les politiques sportives concernent la faisabilité socio-économique puis la gestion des équipements, l'utilisation des activités sportives à des fins d'animation, de prévention ou d'insertion des jeunes en difficulté, l'impact des événements ou encore le développement du sport compétitif et en particulier au plus haut niveau.

Ces approches soulignent aussi la forte dispersion des budgets sportifs, rapportés au nombre d'habitants. L'instabilité des décisions budgétaires reste aussi de règle. D'une année sur l'autre, les budgets sont soumis à des évolutions, positives ou négatives, très marquées. Sauf cas particuliers, la versatilité et la disparité des budgets sportifs attestent de la difficulté à concevoir et à développer des politiques sportives structurées et inscrites dans la durée. Il est vrai que si la question sportive est (faussement) consensuelle, elle est rarement un enjeu local important. En tout cas, ces caractéristiques attestent de la forte dépendance des politiques sportives locales vis-à-vis de la conjoncture économique et du calendrier électoral.

\section{3) Des politiques sportives structurantes}

Les études confirment le rôle décisif des politiques sportives locales dans la structuration du système sportif. Par leur apport financier, les collectivités locales, et en particulier les communes, ont, depuis plusieurs décennies, créé les conditions de la pratique du plus grand nombre en construisant les équipements sportifs, en subventionnant la quasi-totalité des clubs, en recrutant des personnels spécialisés (la constitution de la filière sportive des collectivités locales, en 1992, a permis de recruter des opérateurs, des éducateurs sportifs et des conseillers destinés à diriger les services municipaux des sports) et en permettant l'accès gratuit des publics scolaires (souvent de la maternelle à l'université) aux équipements sportifs dont elles assurent la totalité des coûts de fonctionnement. Par leurs choix politiques, elles ont contribué aussi à modeler les goûts sportifs (en construisant certains types d'équipements ou en organisant telle ou telle manifestation), à accompagner le développement des modes sportives (en adoptant certaines réglementations ou en construisant par exemple les équipements sportifs de proximité), ou encore à structurer le sport de haut niveau. Il est vrai que les élus locaux (plus dans les communes que dans les conseils généraux et régionaux) sont en «première ligne », directement au contact des pratiquants et de leurs groupes de pression, et des non-pratiquants... qui n'en sont pas moins électeurs. 


\section{4) Les collectivités locales arbitrent les concurrences}

Dans le même temps, un processus de diversification des offres sportives s'engageait, mettant les collectivités locales en position d'arbitre. Si le club était traditionnellement le seul porteur de l'offre, il est rejoint par de nouveaux offreurs de pratiques sportives, chacun étant porteur de ses propres valeurs et de ses « manières de faire ». Des entreprises privées se sont intéressées dès les années 1970 aux activités sportives dites « de forme et de remise en forme ». Puis ce mouvement a concerné d'autres activités, comme par exemple l'équitation où une partie des centres équestres est de statut privé. Les communes, en particulier celles engagées dans la lutte contre l'exclusion sociale ${ }^{8}$, ont embauché leurs propres éducateurs sportifs pour développer des animations sportives, d'abord estivales, puis pendant toutes les vacances, puis tout au long de l'année. Ce mouvement a été largement conforté par les dispositifs relatifs à « l'aménagement des rythmes de vie de l'enfant » qui, d'une façon ou d'une autre, ont incité les communes à se préoccuper des temps péri et extrascolaires. Des associations diverses regroupant des femmes, des jeunes, des personnes âgées, des habitants du même quartier ou se constituant sur des bases ethniques, religieuses ou simplement commerciales, se sont positionnées sur le marché des pratiques sportives. Enfin, au-delà de ces concurrences institutionnelles, le mouvement sportif mais aussi les autres offreurs ont été profondément déroutés parce qu'il est convenu d'appeler maintenant les pratiques auto-organisées ${ }^{9}$.

\section{II) Soutien des collectivités au sport professionnel : le temps des choix}

\section{1) Les problèmes méthodologiques de la mesure du financement public du sport professionnel}

Alors que la comptabilité publique semble rechercher l'homogénéité des systèmes d'informations comptables, la réalité des pratiques complique la tâche des chercheurs en management public. La ventilation comptable ne permet pas de distinguer les dépenses liées au domaine «sport » et, dans ce cadre, au seul sport professionnel. De plus, les éléments indirects ne sont généralement pas intégrés. Ainsi la

8. D. Charrier et J. Jourdan, «Pratiques sportives et jeunes en difficulté : 20 ans d'innovations et d'illusions... et des acquis à capitaliser », dans M. FALCOZ et M. KoEBEL (dir.), L'Intégration par le sport, représentations et réalités, Paris, L'Harmattan (à paraître en 2005).

9. G. VIEILlE MARCHISET, Sports de rue et pouvoirs sportifs, Besançon, Presses universitaires franc-comtoises, 2003. 
construction et la mise à disposition d'installation, de personnels, les exonérations fiscales ou les prises en charge de dépenses de fonctionnement courantes ne sont pas traitées selon une doctrine unique, malgré l'insistance des chambres régionales des comptes. L'achat de prestations aux clubs (places au stade, achat de soirées de gala) est parfois imputé au budget communication mais dans certains cas relève de l'action sociale de la collectivité. L'importance de ces aspects indirects, qui abondent fortement le subventionnement officiel, s'est traduit par une limitation formelle imposée par le législateur de ces dépenses dans les décrets d'application de la loi Buffet. Les engagements hors bilan et la consolidation des dettes sont également source de difficulté. Les comptabilités, qu'elles soient publiques ou privées, ont de plus en plus de mal à rendre compte d'éléments non intégrables dans des bilans comptables d'essence patrimoniale.

Si ces biais ne contribuent pas à améliorer la transparence des coûts, ces remarques méthodologiques, malgré les limites qu'elles définissent, n'empêchent toutefois pas de tenter un tour d'horizon des pratiques en matière de financement du sport professionnel.

\section{2) Sport professionnel et collectivités locales : des liens forts et anciens}

Les collectivités ont toujours soutenu les activités sportives d'élite évoluant sur leur territoire. Sur le fond, fédérer les hommes dans leur sentiment d'appartenance, fournir un spectacle à la population ou exalter le dynamisme de la région (et de ses élus) constituent des objectifs fréquemment mentionnés. Sur la forme, construction et entretien d'installations, subventions directes, emplois réservés, mises à disposition, exonérations fiscales, cautionnements, prises de participation dans une société d'économie mixte (SEM) sont autant de moyens de soutenir un champion local ou, plus fréquemment, une équipe fanion. Une autre démarche d'utilisation du sport spectacle consiste à favoriser l'organisation d'événements ponctuels (Jeux olympiques, championnat du monde) ou récurrents (course à la voile, tournoi de tennis, étape du Tour de France).

Les raisons de ce soutien sont multiples et connues. Elles renvoient à la volonté de communiquer en interne et/ou en externe ou encore à la recherche de retombées économiques.

Par ailleurs, le sport professionnel a connu au cours des vingt dernières années une transformation majeure. Suivant l'ensemble de l'économie mondiale dans le processus de déréglementation observé à la fin du $\mathrm{XX}^{\mathrm{e}}$ siècle, les entreprises de spectacle sportif évoluant dans des championnats d'équipes connaissent des phénomènes de concentration et de privatisation dans un marché en croissance. 


\section{3) Politique publique locale et sport spectacle : deux décennies de changement}

L'observation des vingt dernières années offre la possibilité de dégager trois tendances majeures.

Apparaît d'abord une volonté de désengagement public qui débouche sur une dualisation du système. Depuis le début des années 1980, le sport d'élite s'est structuré, comme de nombreux autres secteurs selon un modèle entrepreneurial privé. Le club associatif et ses sociétaires devenaient une entreprise, propriété de ses actionnaires sous l'effet de l'irruption massive de la dimension économique dans le sport devenu spectacle.

Le législateur a enfoncé le clou en rendant obligatoire le passage à une structure privée commerciale dès le franchissement de seuils d'activité. Complétant cette volonté, le décret dit « Pasqua » avait en 1994 engagé un processus de disparition de toutes les aides publiques locales aux clubs professionnels. Devant l'impossibilité d'obtenir ce résultat, la loi sur le sport (2000) et le décret sur les aides publiques (2002) ont finalement limité - de manière discutable dans le principe - la liberté des élus ${ }^{10}$. Ainsi, le montant maximum des prestations de services achetées par les collectivités locales est de 1,6 milliard d'euros alors que les subventions directes ne peuvent dépasser 2,3 milliards d'euros. Ces dernières doivent se limiter aux seules dépenses de sécurité dans le stade, aides au centre de formation et pour des actions d'insertion sociale par le sport.

Comme le montre le tableau I, le processus est allé, apparemment, à son terme.

Tableau I

Statuts juridiques des clubs de football en France

(1990-2003)

\section{Illustration non autorisée à la diffusion}

Source : LFP.

Toutefois, cette évolution voulue par le politique vers des «clubs privés autonomes » s'est heurtée à une difficulté majeure : les entreprises de spectacle spor-

10. C. Durand et E. Bayle, «Financement des entreprises de spectacle sportif par les collectivités locales : quelle liberté pour les élus ? », dans R. LE DUFF et J.-J. RigaL (dir.), Démocratie et management local, Paris, Dalloz, 2001. 
tif ne peuvent se passer des aides publiques locales. Le système s'est finalement scindé en deux sphères : les privatisées et les «pseudo »-privatisées. Le tableau II développe le montant des aides publiques directes aux équipes françaises.

\section{Tableau L}

Subventions publiques directes aux clubs professionnels (hors coûts indirects)

(en millions d'euros)

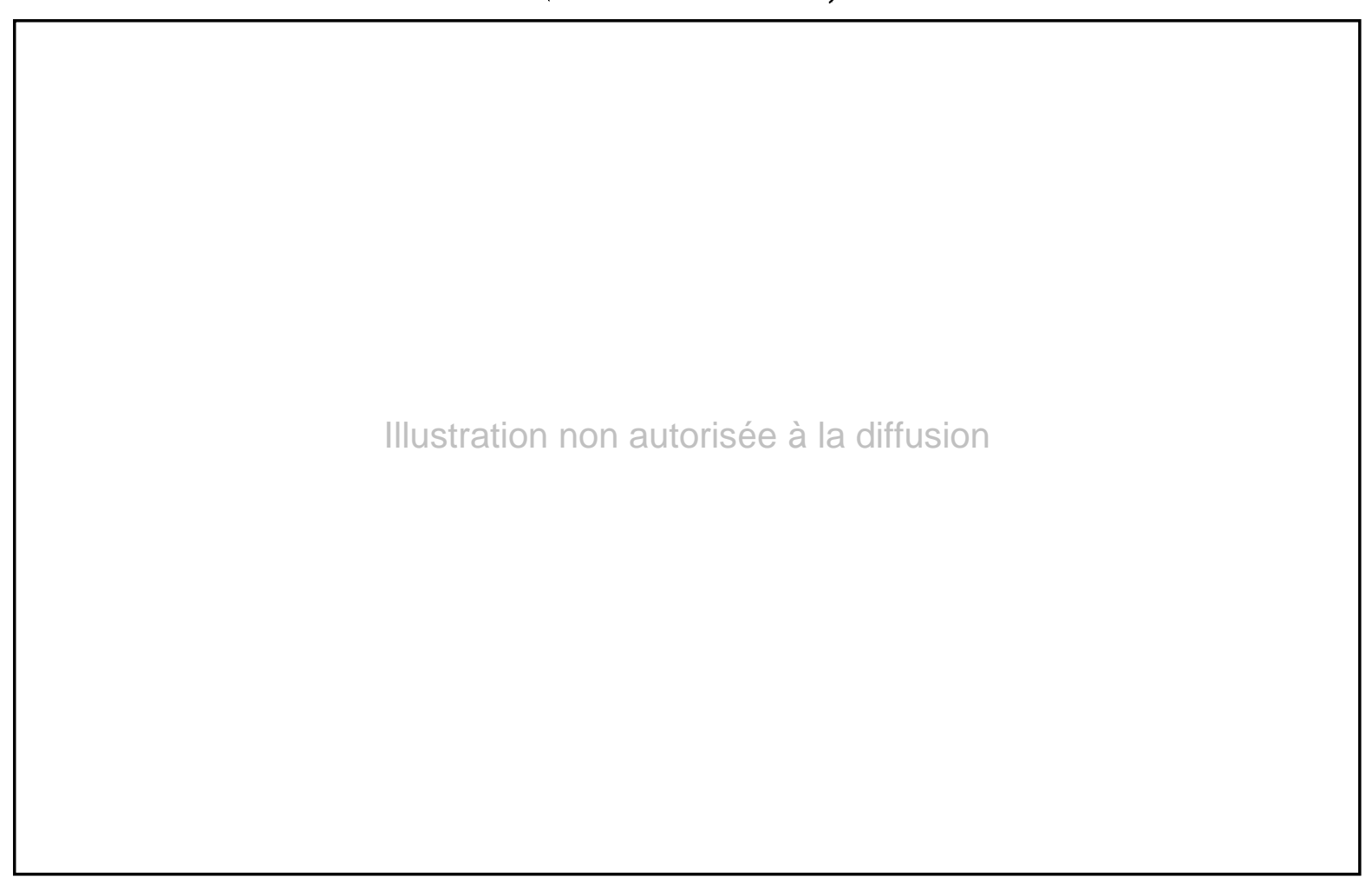

Source: LFP, LNB et Denis (2004).

En pro A de basket, l'aide moyenne est stable autour de 1 million d'euros par club, soit environ $30 \%$ de ses recettes. Les équipes de pro B dépendent encore plus largement des collectivités locales avec une part qui oscille entre 50 et $57 \%$ des budgets (le montant passant de 0,5 million d'euros en 1999-2000 à 0,7 million d'euros par club en 2002-2003). La Ligue féminine de basket bénéficie de 3,75 millions d'euros d'aides publiques, soit $62 \%$ des recettes et 375000 euros par équipe en moyenne.

En rugby, la Ligue nationale de rugby annonce que les 16 équipes du top 16 bénéficiaient de $10 \%$ de leurs recettes 2001-2002 sous forme de subventions directes, soit environ 8 des 100 millions d'euros de budget total soit une moyenne de 500000 euros par équipe. Ce taux devait passer à $8 \%$ en 2002-2003. En pro D2, le taux de financement public direct était de $28 \%$ en 2001-2002 pour passer à $22 \%$ la saison suivante. Soit environ 8 millions d'euros sur les 34 du total de ce championnat, soit 500000 euros Ces montants ne « tiennent pas compte des prestations payées par les collectivités qui sont classées en sponsoring ».

En handball, la Ligue nationale de handball estime que les subventions représentent $60 \%$ des recettes des 14 clubs de D1, soit 830000 euros en moyenne. En D2, les 333000 euros par équipe représentent $68 \%$ des recettes des 16 clubs.

La Ligue nationale de volleyball estime que les subventions représentent près de $72 \%$ des recettes 2001-2002 des clubs en pro A comme en pro B. Dans un contexte de hausse des budgets, cela représente respectivement 8,6 millions d'euros et 2,9 millions d'euros, soit 600000 euros par équipe de pro $A$ et 200000 euros en pro B. 
Le retrait des collectivités n'est ainsi réel que dans le cas du football et du rugby de premier niveau (ligue 1 et top 16). Avec 4 et $10 \%$ de leurs recettes sous forme d'aides publiques, ces championnats se révèlent assez indépendants dans leur financement. Même en intégrant les soutiens indirects, la plupart de ces équipes ont opéré leur mutation. L'explosion des recettes liées aux droits de retransmissions télévisées et la rénovation des stades à la suite de la Coupe du monde 1998 ont permis une large émancipation. Implantées dans de grandes villes, ce qui dilue l'effort des contribuables, participant régulièrement à des compétitions continentales, ces firmes bénéficient d'une exposition plus importante.

Mais derrière ces quelques clubs-entreprises disposant de ressources privées (public, entreprises, télévisions) suffisantes, la sphère publique ne peut se désengager réellement, tant son retrait entraînerait de difficultés. Ainsi, au cours de la décennie beaucoup de clubs professionnels ont constaté qu'ils ne pouvaient survivre sans aide publique locale, voire nationale via les dispositifs d'aides à l'emploi qui alimentent ces structures en athlètes modestement rémunérés par le contribuable.

Ainsi, le système apparaît aujourd'hui dualisé. D'une part, des clubs où les collectivités, partenaires indispensables, sont «noyées » dans les financements privés. Ces firmes ont facilement trouvé des repreneurs privés. De l'autre, des clubs dits «professionnels » mais dont la dépendance aux contribuables locaux est majeure. Ces sociétés peinent à trouver des actionnaires solvables, la collectivité continuant à jouer en cas de difficultés le rôle de « pompier ».

La seconde tendance concerne l'importance du potentiel local. Il convient de souligner ici une évolution majeure de la doctrine des élus en termes de soutien public aux clubs : une équipe professionnelle apparaît de plus en plus comme un équipement urbain dont l'existence dépend de la zone de chalandise disponible.

L'accession au championnat s'effectue en France, jusqu'ici, selon des critères sportifs : le (ou les) meilleur(s) sportivement accède(nt) à la division supérieure ou aux coupes européennes, les moins bons « descendent ». Ce système de promotion-relégation subit aujourd'hui de nombreuses critiques. L'instabilité générée a vu ses conséquences augmenter du fait du creusement de l'écart entre les ressources et la médiatisation des divisions : installations surdimensionnées pour les uns, «petites » équipes promues mais incapables d'atteindre le niveau sportif et faisant chuter l'intérêt de la compétition pour redescendre plus ou moins rapidement en division inférieure.

La multiplication des faillites en partie liées à ces variations de ressources suscite la réflexion dans toutes les ligues européennes. La généralisation de critères économiques dans l'accès aux ligues, voulue par les investisseurs, aboutit pour les collectivités locales à une adaptation de leur politique sportive à la masse démographique disponible. Un championnat de sport collectif apparaît aujourd'hui comme un réseau de contributeurs à une prospérité commune. Chacun des membres de la ligue doit amener un marché potentiel en phase avec l'apport des autres membres du championnat.

Ce constat n'est pas sans conséquence stratégique pour les élus : un stade de football de 20000 places coûte environ 25 millions d'euros, hors foncier, quid de cet 
équipement en cas de relégation? Dans le cas des aires urbaines françaises, on observe qu'une typologie hiérarchique et sportive se dégage aujourd'hui nettement.

Tableau III

Clubs professionnels et aires urbaines

(2003-2004)

Étude menée sur les aires urbaines française pour la saison 2003-2004 avec les sports et les niveaux suivants : football (D1, D2, N1), basket (pro A, pro B et Ligue féminine), hand et volley (D1), hockey (Élite), Rugby (top 16 et D2) pour un total de 179 clubs.

C. Durand, L. Ravenel et B. Helleu, Basket professionnel français: approche stratégique et géomarketing, Voiron, Presses universitaires du Sport (à paraître en 2005).

À moins de 100000 habitants, la présence d'une équipe est rare. Quand cela se produit, la monoactivité sportive est obligatoire compte tenu des moyens financiers disponibles et des potentiels de population. Au-dessus de 250000 habitants, le nombre ainsi que la diversité des disciplines augmentent. Le problème concerne les villes « moyennes ». De 100000 à 250000 habitants, l'absence de clubs devient plus rare, mais la norme reste la monoactivité. Deux disciplines sont envisageables mais à condition d'abaisser les niveaux sportifs. Conscients de cette contrainte, de nombreux élus ont opté au cours des années 1990 pour un soutien exclusif et ciblé, après avoir longtemps pratiqué la diversité sportive.

La troisième tendance est la pérennisation du financement public local mais la fin de la tentation de cogestion des clubs. Si pour une majorité des cas, le soutien massif des collectivités est resté nécessaire, la nature du lien entre les clubs et la sphère publique locale a changé. Alors que les associations s'appuyaient formellement sur la cité, le changement de statut s'est accompagné d'un changement de relations. Cette tendance est symbolisée par la disparition des sociétés d'économie mixte alors que les années 1980 avaient avancé l'idée d'une participation "naturelle » des collectivités locales à la gestion des clubs. Il est vrai que certaines expériences de SEM n'ont ainsi pas été aussi heureuses que le souhaitaient les élus. L'importance des 
pertes supportées par les collectivités dans les SEM ou dans les associations au début des années 1990 a sonné le glas de la cogestion ".

Cette transformation de logique s'est faite sous la double pression de l'environnement - privatisation du secteur - mais aussi du législateur qui a précipité ce retrait par les différents textes votés à partir de 1989 (Pasqua, Bredin, Buffet). Cette forme de régulation autoritaire se retrouve dans toute la doctrine publique depuis 1992 : cautions interdites, obligation de création dans les ligues d'instances de contrôle financier (DNCG en football, CCG en basket), limitation des subventions et des achats de prestations, interdiction des SEM sportives.

\section{III) Les politiques sportives locales dans l'avenir}

Depuis une vingtaine d'années, un processus de rationalisation a été engagé grâce à la montée des enjeux sportifs, économiques, sociaux et politiques et des compétences dans les services municipaux. Cet « effet d'apprentissage » a d'abord concerné les équipements : ils sont devenus plus économes en énergie, plus accessibles et surtout mieux adaptés aux besoins des pratiquants. Un véritable effort a été entrepris au niveau de la gestion des services municipaux et en particulier en ce qui concerne l'attribution des subventions, la formation des personnels, les stratégies d'intervention éducative et sociale ou l'organisation des événements sportifs. Au niveau des sports professionnels, les collectivités, qui découvraient une certaine autonomie dans les années 1980, ont aussi changé de stratégie. L'irruption d'opérateurs privés, parfois difficiles à convaincre, a eu un double effet. D'une part, elle a permis à certaines collectivités de se désengager de la gestion de leur club. D'autre part, le fossé créé entre « gros » et « petits » a entraîné la mise en ouvre de stratégies de différenciation à travers des choix adaptés aux moyens locaux. Ce phénomène, observé partout en Europe, a conduit à une réduction drastique du nombre de clubs professionnels. Les populations, déjà sensibles à la redistribution spatiale d'un certain nombre de services publics liés à la santé, à l'éducation ou au transport vivent parfois difficilement ces évolutions.

$\mathrm{Si}$, au-delà des évolutions conjoncturelles, les caractéristiques du financement du sport par les collectivités locales paraissent fixées, la situation pourrait changer dans les prochaines années sous l'influence du développement de l'intercommunalité sportive, de la montée des compétences dans le mouvement sportif, de la nécessité de réhabiliter de très nombreux équipements sportifs ou encore de l'évolution du sport professioninel.

11. C. Durand, «Sport professionnel et collectivités locales : la fin d'une idée, l'économie mixte... Bilan d'un quart de siècle », dans G. CliQuet et G. ORANGE (dir.), Mélanges en l'honneur du professeur Le Duff, Rouen, Presses universitaires de Rouen, 2002. 
Finalement, à travers le débat sur le financement du sport local par le contribuable, c'est le statut sociétal du sport qui reste à définir.

Soit cette activité est à soutenir car d'intérêt général au même titre que d'autres activités culturelles, et la sphère publique est dans son rôle en intervenant. Insertion, pacification sociale, promotion d'un sentiment identitaire, amélioration de l'état de santé de la population ou encore développement économique local justifient une intervention massive, éventuellement autoritaire, du politique qui dans ce contexte refuse de transférer sa compétence au secteur privé, au «tout économique ».

Soit le sport et en particulier celui « qui se regarde» est considéré comme essentiellement économique et relève alors de la sphère privée. Dans cette vision, les collectivités locales n'interviennent que dans un cadre très limité, celui d'un soutien classique aux entreprises locales, quels que soient leurs secteurs d'activité. Sur ce plan, notre pays n'a pas clairement défini de règles, chacun des acteurs agissant dans son propre cadre. De ce débat sur le statut même de l'activité sportive professionnelle, qui est certes économique mais qui n'est peut-être pas que cela, sortiront des règles du jeu rénovées qui permettront éventuellement le maintien de cette activité partout dans le pays et pour toutes les populations.

Les changements attendus, mais aussi les ruptures imprévisibles, devraient inciter les politiques sportives à évoluer radicalement sous les nouvelles pressions de l'environnement. Les acteurs sportifs pourront-ils se mobiliser pour anticiper et pour participer à cette nouvelle construction et non la subir ? Il s'agit bien d'imaginer d'autres perspectives, bien au-delà du vieux débat « sport d'élite ou sport de masse » puisque les politiques sportives peuvent devenir de véritables leviers des politiques économiques et sociales, et constituer un outil essentiel de la gestion des territoires et des populations.

Restera alors à définir les moyens d'intégrer le sport dans le projet local entre renforcement, en réaffirmant la spécificité du sport comme outil de développement local, et dilution, par la soumission du sport à d'autres politiques sectorielles locales. 